\title{
A Comprehensive Study of Palmprint based Authentication
}

\author{
Madasu \\ Hanmandlu \\ I.I.T. Delhi, \\ New Delhi-110016, \\ India
}

\author{
Neha Mittal \\ J.P. Institute of \\ Engineering \\ \&Technology, \\ Meerut, U.P. \\ India
}

\author{
Ankit Gureja \\ Jamia Milial Islamia \\ Delhi \\ New Delhi
}

\author{
Ritu Vijay \\ Banasthali University, \\ Banasthali, \\ Rajasthan,India
}

\begin{abstract}
This paper presents some new features for the palmprint based authentication. The Region of interest (ROI) is extracted from the palmprint image by finding a tangent to the curves between fingers. The perpendicular bisector of this tangent and the tangent itself help demarcate the rectangular area that forms the ROI of the palmprint. Four approaches are presented for the feature extraction. In the first approach the ROI is divided into a suitable number of non-overlapping windows from which fuzzy features are extracted. In the second approach multi-scale wavelet decomposition is applied on the ROI and the detail images are combined to yield a composite image which is partitioned into non-overlapping windows and energy features are extracted. In the third approach sigmoid features are extracted from the ROI and in the fourth approach feature extraction is done using Local Binary Pattern (LBP) based on the directional gradient response. These four sets of features are used for the authentication of users from two databases using Euclidean Distance, Chi square measure and Support Vector Machines as classifiers.
\end{abstract}

\section{General Terms}

Palmprint authentication, ROI, feature extraction, matching,

\section{Keywords}

Fuzzy features, Wavelet features, sigmoid feature, Local Binary Pattern, Support Vector Machines.

\section{INTRODUCTION}

The palmprint as a biometric modality is slowly but decisively gaining acceptance in the field of biometrics. As compared to other biometric modalities, it is bestowed with enormous information that is a boon of its discriminating power. We will discuss a few important contributions made on this modality.

A palmprint authentication system in [1] is specifically designed to overcome the limitations of the contemporary biometric authentication systems. In this system, geometric and pseudo Zernike moments are employed as feature extractors from the decomposed palmprint image. Before moment computation, wavelet transform is applied to decompose a palmprint image into lower and higher frequency subbands. This decomposition reduces the computational burden of the moment calculations drastically. The generated wavelet moments based features are used to create a cancelable verification key with a set of random data. This private binary key can be canceled and replaced. Besides this key also possesses high data capture offset tolerance, with highly correlated bit strings for intra-class population. This property allows a clear separation of the genuine and imposter populations as well as attains the zero Equal Error Rate attainment which cannot be realized by the conventional biometric based authentication system.

Matching of palmprints in [2] deals with the feasibility of person identification based on a set of feature points extracted along the prominent palm lines (and the associated line orientation) from a given palmprint image. Next a decision is made whether two palmprints belong to the same hand by computing a matching score between the corresponding sets of feature points of the two palmprints. Two sets of features/orientations are matched using point matching technique which takes into account the nonlinear deformations as well as the outliers present in the two sets. The estimates of the matching score distributions for the genuine and imposter sets of palm pairs indicate that palmprints have a good discrimination power.

Palmprint Verification with moments in [3] introduces an experimental evaluation of the effectiveness of utilizing three well known orthogonal moments, namely Zernike moments, pseudo Zernike moments and Legendre moments for palmprint verification. The idea of implementing orthogonal moments as palmprint feature extractors is prompted by the fact that the principal features of palmprint are based on the line structure. These orthogonal moments are able to define statistical and geometrical features containing line structure information about palmprint. Experimental results show that the performance of the system is dependent on the moment order as well as the type of moments. The orthogonal property of these moments is able to characterize independent features of the palmprint image and thus have minimum information redundancy in a moment set. Palmprint verification using complex wavelet transform [4] moots a modified complex wavelet structural similarity index (CW-SSIM) as the matching score for identifying the input palmprint. The local structure information of a palmprint is hidden in the relative phase patterns of the complex wavelet coefficients and a constant phase shift of all coefficients does not change the structure of local image features. Since CW-SSIM is robust to translation, small rotation and distortion, a fast rough alignment of palmprint images is sufficient. CWSSIM is also in sensitive to luminance and contrast changes.

Palmprint classification in [5] is an important indexing mechanism in a palmprint database. This algorithm uses a novel representation and is based on two-stage classifier that provides even-distributed categories. A novel representation scheme is directly derived from the principal line structures. 
The representation does not use wrinkles, and singular points. It is capable of tolerating poor image quality.

In this paper, we will explore feature extraction using Local Binary Pattern Operator, fuzzy features and wavelet features extracted from a palmprint. Though Gabor features have been extensively used on palmprints, it is observed that they are sensitive to slight changes in size and orientation of a palm. An effort is made to work on two counts: An efficient extraction of Region of Interest (ROI) and an effective features election.

The organization of the paper is as follows: Section 2 presents the extraction of ROI followed by the extraction of wavelet, fuzzy, sigmoid and LBP features. Matching and results of implementation are described in Sections 3 and 4 respectively. Finally conclusions are drawn in Section 5.

\section{FEATURE EXTRACTION}

\subsection{Extraction of ROI}

The procedure for the extraction of ROI from a palmprint is adopted from [6]. It is implemented on both IITD and PolyU databases in [7]-[8]. A sample of the extracted ROI of palmprint is shown in Fig. 1.
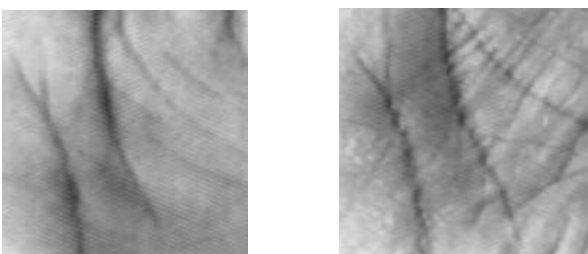

Fig 1: The extracted ROI of palmprint

Our methodology starts with the partitioning of ROIs into sub images to fit the fuzzification functions like sigmoid on them. Wavelet and fuzzy features from [7] are described briefly.

\subsection{Wavelet Features}

Wavelets are immensely suitable for texture analysis. As a palmprint is a rich source of texture, we are inclined to investigate the effectiveness of Haar wavelets on the palmprints. Simplicity of these wavelets is an overriding factor in the choice of these wavelets though other celebrated wavelets such as Daubechies wavelets, Symlets are also tried but the improvement accrued from the latter is not commensurate with the effort.

A two-dimensional discrete wavelet transform is applied on the ROI of a palmprint resulting in four subbands: HL1 $\left(\mathrm{H}_{1}\right)$, LH1 $\left(\mathrm{V}_{1}\right)$ and HH1 $\left(\mathrm{D}_{1}\right)$, which constitute the detail components and LL1 $\left(\mathrm{A}_{1}\right)$, which is the approximation component at the first level of decomposition. Two-level decomposition of ROI using 2D Discrete Wavelet Transform (DWT) is shown in Fig. 2. Note that $\mathrm{H}_{1}, \mathrm{~V}_{1}$ and $\mathrm{D}_{1}$ are aggregated to get a composite detail image at the first decomposition. The detail images at the $\mathrm{k}^{\text {th }}$ decomposition are denoted by $H_{k}, V_{k}, D_{k}$. Each detail image represents a certain high frequency structure, i.e. lines, creases, wrinkles, ridges on a palmprint. Fig. 3 shows the three-level decomposition. The approximation image $\mathrm{A}_{1}$ can be further decomposed into four sub bands and the process is repeated till the information from $A_{n}$ at the nth decomposition is insignificant.

Here, a palmprint image is decomposed into four time scales using Haar wavelets beyond which results are found to be deteriorating. In any decomposition, the detail images include horizontal, vertical and diagonal parts of the decomposed image. These three detail images are superimposed to yield composite mage. It may be noted that as the decomposition level increases the size of the detail images also decreases. The results of authentication correspond to the composite detail image.

As we have four decompositions there will be five superimposed detail images called composite detail images of different sizes: $1^{\text {st }}, 2^{\text {nd }}, 3^{\text {rd }}$, and $4^{\text {th }}$ detail images. Barring the first as the wavelet features derived from it are insignificant, other three composite detail images $S_{k}, k=2, . ., 4$ are divided into non overlapping windows [19]. The $\mathrm{k}^{\text {th }}$ composite detail image is computed from:

$$
S_{k}(i, j)=H_{k}(i, j)+V_{k}(i, j)+D_{k}(i, j)
$$

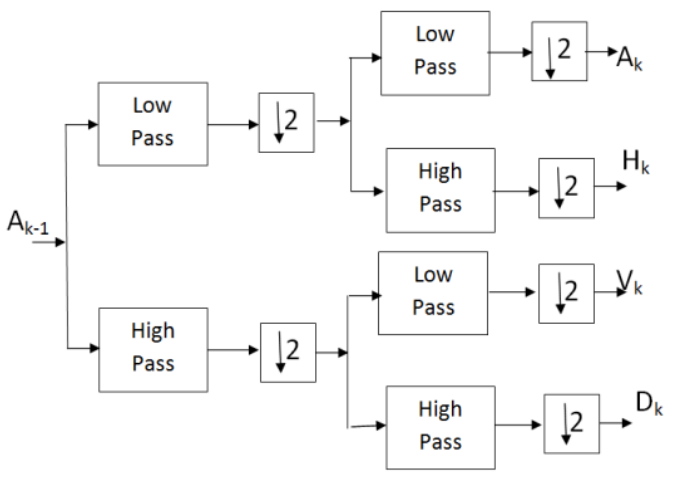

Fig 2: Two-level decomposition using 2D DWT

The wavelet feature for $l^{\text {th }}$ window of $\mathrm{k}^{\text {th }}$ composite detail image is found from:

$E_{k}(l)=\sum_{i=1}^{w} \sum_{j=1}^{w}\left(S_{k}(i, j)\right)^{2}$

where $H_{k}, V_{k}$ and $D_{k}$ are the detail images in horizontal, vertical and diagonal directions respectively.

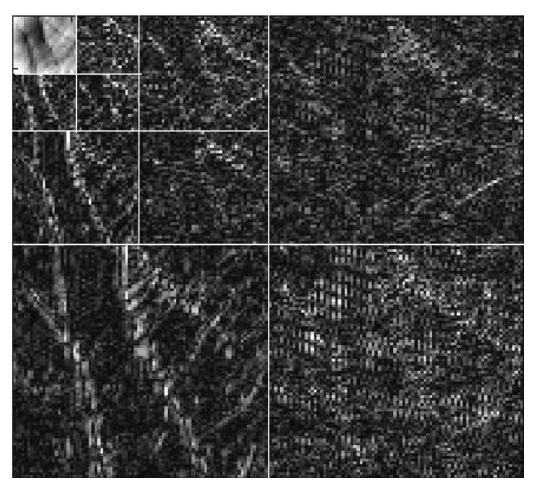

Fig. 3: Three-level DWT decomposition

\subsection{Fuzzy Features}

The palmprints possess random textures consisting of zigzag and crisscrossing lines in addition to three principal lines. We are mainly interested in capturing the random texture. One way is to partition the ROI of palmprint into windows of appropriate size and represent the information by fuzzy features with respect to the centre of the window. The fuzzy features of the neighborhood are aggregated to yield a representative value called cumulative response that 
represents the texture. The steps involved in the Fuzzy features are given as part of the algorithm here.

\section{An Algorithm for Feature Extraction}

1. Divide the ROI into a fixed number of non-overlapping windows.

2. Compute the average intensity $\mathrm{I}_{\text {avg }}$ in a window of size $w x w$ using

$$
I_{\text {avg }}=\frac{1}{W x W} \sum_{i=1}^{W} \sum_{j \neq 1}^{W} I(i, j)
$$

3. Determine the maximum intensity $I_{\max }$ in the window.

4. Compute $\mu_{\mathrm{ij}}$ for every pixel in the widow using

$$
\mu_{i j}=1-\left(\frac{\left|I(i, j)-I_{a v g}\right|}{I_{\max }}\right)
$$

5. Find the cumulative response for $l^{\text {th }}$ window from:

$$
F(l)=\frac{\sum_{i=1}^{W} \sum_{j=1}^{W} \mu_{i j} . I(i, j)}{\sum_{i=1}^{W} \sum_{j=1}^{W} \mu_{i j}}
$$

The above value is used as a feature and the number of these features is equal to the number of windows in a ROI.

\subsection{Sigmoid Features}

The steps for the extraction of sigmoid features are as follows:

1. Divide the ROI into a fixed number of non-overlapping windows.

2. Compute the average intensity $I_{\text {avg }}$ in a window of size wxw using (3)

3. Find the maximum intensity, $I_{\max }$ in the window and compute the membership function using (4).

4. Compute the sigmoid function for $l^{\text {th }}$ window from:

$$
\operatorname{Sig}(l)=\sum_{i=1}^{W} \sum_{j=1}^{W} \frac{I_{a v g}}{1+\exp \left(-\mu_{i j}\right)}
$$

\subsection{Local Binary Pattern (LBP) Feature}

The Sobel compass operator [9] is applied on ROI in eight directions to find edges. The results of application of Sobel operator are displayed in Fig. 4 and the eight Sobel masks are shown in Fig. 5.
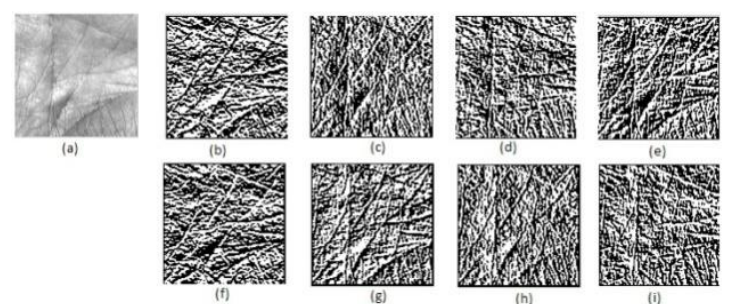

Fig 4: The directional responses of Sobel masks. (a) Original palmprint, (b) to (i) responses in the eight directions
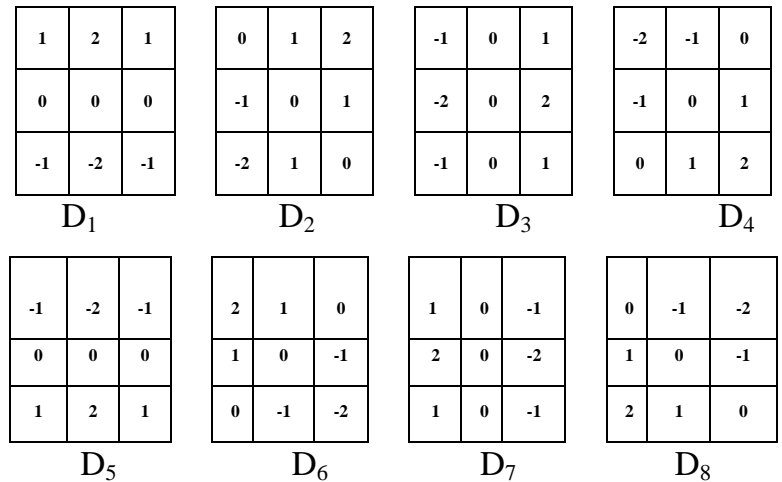

Fig 5: The eight 3x3 Sobel masks

\section{Local Binary Patterns (LBP)}

The LBP operator due to Ojala [10] is employed here for the texture analysis. This operator assigns a binary value to every pixel in the eight-neighborhood by thresholding its gray level with respect to the gray level of center pixel. The feature vector is formed by concatenating the binary bits in the anticlockwise direction, shown in Fig. 6.

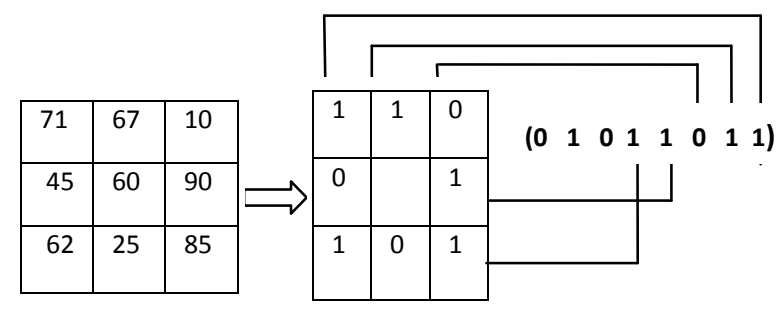

Fig 6: Obtaining a Binary label for a pixel

As LBP code is invariant to any monotonic transformation on the pixel values, it is suitable to represent the palms that are illuminated from a light source of varying intensity but at fixed distance.

An LBP string is termed uniform if it is made up of at most 2bit transitions from 0 to 1 or vice-versa. For example, 11111110 and 11101111 are uniform LBP strings whereas 11101011 and 10110101 are non-uniform. There are 58 labels of uniform patterns and the rest 198 labels are of non-uniform, all of which are assigned to $59^{\text {th }}$ label. Thus we have a set of 59 LBP patterns labeled by $\{1,2, \ldots, 59\}$. Having the labels handy, a histogram of labels can be constructed as:

$h_{l}=\sum\{L(i, j)=l\}, l=1,2 \ldots . .59$

The histogram of labels is used as the texture descriptor that represents the local characteristics of the image. Following the approach in [11] the palm print images are divided into $\mathrm{N}$ equal sub-windows and the texture descriptor of each subwindow is formed from the average of all the directional responses by the application of the Sobel masks. These local texture descriptors are then concatenated to form the global texture descriptor. Thus, the texture descriptor for a given palm print is of size $\{59$ (no. of labels) $\times \mathrm{N}$ (no. of subwindows) $\}$.

\section{MATCHING}

\subsection{Euclidean Distance Classifier}

Given two data sets of features corresponding to the training and testing samples, a matching algorithm determines the degree of similarity between them. A Euclidean distance is 
adopted as a measure of dissimilarity for the palmprint matching using both wavelet and fuzzy features.

\subsection{Chi-square Measure}

In the case of LBP, the matching of an image pair is done by computing the distance between the two LBP feature histograms of training and test samples. The larger the distance between the histograms the more dissimilar are the images. The Chi-Square distance between the two histograms $\mathrm{S}$ and $\mathrm{M}$ can be defined as:

$$
\chi^{2}(S, M)=\sum_{b=1}^{B} \frac{\left(S_{b}-M_{b}\right)^{2}}{S_{b}+M_{b}}
$$

Where $S_{b}$ and $M_{b}$ are normalized enhanced histograms; the index $b$ refers to the $b^{\text {th }}$ bin of the histogram, which in this case varies from 1 to 59 .

\subsection{Support Vector Machine}

SVM based on the principle of Structural Risk Minimization (SRM) [12]-[13] constructs a set of hyper-planes in a high dimensional space for the classification of input features. Considering a two-class problem to be solved by a SVM, we start with a training sample described by a set of features $\mathrm{x}_{\mathrm{i}} \in \mathrm{R}^{\mathrm{n}}, \mathrm{n}=1,2, \ldots, \mathrm{N}, \mathrm{N}$ being the number of features belong to one of two classes indicated by the label $y, \in\{+1,-1\}$. The data to be classified by the SVM may not be linearly separable in its original domain. In the linearly non-separable case the data is projected onto a higher dimensional feature space using Kernel functions [14] defined as:

$$
K\left(x_{i}, x_{j}\right)=\varphi\left(x_{i}\right)^{T} \cdot \varphi\left(x_{j}\right) \quad \mathrm{i}, \mathrm{j}=1,2,3, \ldots \ldots \mathrm{N}
$$

where $\varphi$ is the function that maps the original data onto the higher dimensional space. The SVM now generates a hyperplane in this space with the decision boundary defined as:

$$
f(x)=\sum_{i=1}^{N} y_{i} \alpha_{i} K\left(x, x_{j}\right)+b
$$

Where $\alpha_{i}$ is the non-negative Lagrange multipliers subjected to a quadratic optimization problem.

Minimize:

$$
\begin{aligned}
& L(w, b, a)=\sum_{i=1}^{n} \alpha_{i}-\frac{1}{2} \sum_{i=1}^{N} \sum_{j=1}^{N} y_{i} y_{j} \alpha_{i} \alpha_{j} K\left(x_{i}, x_{j}\right) \\
& \text { Subject to: } 0 \leq \alpha_{i} \leq C_{i}, i=1,2,3 \ldots . N
\end{aligned}
$$

where the cost parameter, $\mathrm{C}$ controls the trade-off between the training errors and the rigid margins. The two kernel functions used in this work are :

Linear kernel:

$$
K\left(x_{i}, x_{j}\right)=a \cdot x_{i}^{T} \cdot x_{j}+b
$$

Polynomial kernel function:

$$
K\left(x_{i}, x_{j}\right)=\left(\alpha x_{i}^{T} x_{j}+c\right)^{d}
$$

In the above the adjustable parameters are slope $(\alpha)$, constants $\mathrm{a}, \mathrm{b}, \mathrm{c}$ and the degree of polynomial $\mathrm{d}$ which is varied in the polynomials kernel function. For the classification of the data LIBSVM [15] is pressed into service. The values of the parameters are fixed at: $\mathrm{a}=1, \mathrm{c}=0, \mathrm{~T}=1$ and $\mathrm{d}=1,2$ and 3 .

\section{RESULTS OF IMPLEMENTATION}

\subsection{Palmprint Database}

The study is conducted on two databases. The first database is from PolyU [16], which consists of palm-print images from 193 individuals out of which 131 are males with the age distribution: younger than 30 years old constitute about $86 \%$, and older than 50 years constitute about $3 \%$. The database was collected on two different occasions, the average time interval between the two being 69 days. On each occasion, 10 images from the left and the right hands were taken from the individuals. Hence the database consists of 7752 images from 386 users.

The second database is taken from Biometrics Research Laboratory, IIT Delhi. This database consists of more than 100 users in the age group of 17-60. The images were acquired online using a PC connected to the Canon A630 digital camera. The database consists of a minimum of 7 images per user per hand and the size of the images is $768 \mathrm{x}$ 576 pixels with a resolution of $96 \mathrm{dpi}$.

\subsection{Discussion of Results}

Feature Extraction is undertaken from ROIs of palm images. The IITD database consists of 125 users with 5 samples per user totaling $125 \mathrm{X} 5=625$ images. The PolyU database we used 6 samples for each user. Several experiments are conducted by taking different combinations of training and testing samples. For the extraction of the wavelet, fuzzy and sigmoid features, the size of a window is also varied whereas in the LBP based feature extraction, the whole image is divided into $\mathrm{N}$ sub-windows to see its effect on the scores.

The recognition rates corresponding to Wavelet features from three composite detail images $(\mathrm{S} 4, \mathrm{~S} 3, \mathrm{~S} 2)$ fuzzy features and sigmoid features on different window sizes using Euclidean classifier and SVM classifier are shown in Tables 1-5 for the IITD database and in Tables 7-11 for the PolyU database. Tables 1-3 give the results of wavelets and Table 4 corresponds to fuzzy features and Table 5 to sigmoid features. Tables 7-9 give the results of wavelets. Table 10 corresponds to fuzzy features and Table 11 to sigmoid features. In these tables, there are two entries. The first entry gives the validation result whereas the bracketed entry gives the crossvalidation result. The recognition rates corresponding to the LBP features for $\mathrm{N}$ sub-windows of size $3 \times 3$ are shown in Tables 6 for the IITD database and in Table 12 for the PolyU database. These results correspond to two compositions of the training and the testing samples (4:1 and 3:2).

Fuzzy, Sigmoid and LBP features have neck to neck competition but wavelet features have slightly inferior performance. The cross validation results can also be seen in the tables in the brackets and the results of cross validation are almost the same as those of the validation.

Table 1. Wavelet Feature (S4) on IITD database

\begin{tabular}{|l|l|l|l|l|}
\hline \multirow{2}{*}{ win size } & \multirow{2}{*}{ ED } & \multicolumn{3}{|c|}{$\begin{array}{c}\text { SVM using different Polynomial } \\
\text { Kernel functions }\end{array}$} \\
\cline { 1 - 3 } $\mathbf{( 4 : 1 )}^{*}$ & & $\mathbf{1}$ degree & $\mathbf{2}$ degree & 3 degree \\
\hline $\mathbf{9 x 9}$ & 97.6 & $99.2(98.4)$ & $97.6(97.6)$ & $96(92)$ \\
\hline $\mathbf{7 x 7}$ & 98.4 & $100(99.2)$ & $98.4(99.2)$ & $96.8(96.8)$ \\
\hline $\mathbf{5 x 5}$ & 100 & $100(99.2)$ & $98.4(99.2)$ & $96.8(97.6)$ \\
\hline$(3: 2)^{*}$ & & & & \\
\hline $\mathbf{9 x 9}$ & 96.4 & $97.6(94.8)$ & $93.6(93.2)$ & $91.2(89.2)$ \\
\hline $\mathbf{7 x 7}$ & 98.4 & $99.2(95.6)$ & $96(94.8)$ & $99.2(92)$ \\
\hline $\mathbf{5 x 5}$ & 99.2 & $98.8(96.4)$ & $96.8(94.8)$ & $94(91.6)$ \\
\hline
\end{tabular}


Table 2. Wavelet Feature (S3) on IITD database

\begin{tabular}{|l|l|l|l|l|}
\hline \multirow{2}{*}{$\begin{array}{c}\text { window } \\
\text { size }\end{array}$} & \multirow{4}{*}{ ED } & \multicolumn{3}{|c|}{$\begin{array}{c}\text { SVM using different Polynomial } \\
\text { Kernel functions }\end{array}$} \\
\cline { 1 - 3 } $\mathbf{( 4 : 1 )}^{*}$ & & 1 degree & 2 degree & 3 degree \\
\hline $\mathbf{9 x 9}$ & 97.6 & $99.2(98.4)$ & $99.2(96.8)$ & $96.8(90.4)$ \\
\hline $\mathbf{7 x 7}$ & 98.4 & $100(99.2)$ & $99.2(99.2)$ & $98.4(96.8)$ \\
\hline $\mathbf{5 x 5}$ & 100 & $100(99.2)$ & $99.2(99.2)$ & $98.4(98.4)$ \\
\hline $\mathbf{( 3 : 2 )}$ & & & & \\
\hline $\mathbf{9 x 9}$ & 97.6 & $98.8(95.2)$ & $96.8(93.6)$ & $93.5(90)$ \\
\hline $\mathbf{7 x 7}$ & 98.8 & $99.2(95.6)$ & $96.8(94.8)$ & $92.4(91.2)$ \\
\hline $\mathbf{5 x 5}$ & 99.2 & $99.2(95.6)$ & $96.8(94)$ & $94(90.4)$ \\
\hline
\end{tabular}

Table 3. Wavelet Feature (S2) on IITD database

\begin{tabular}{|l|l|l|l|l|}
\hline \multirow{2}{*}{$\begin{array}{c}\text { window } \\
\text { size }\end{array}$} & \multirow{2}{*}{ ED } & \multicolumn{3}{|c|}{$\begin{array}{c}\text { SVM using different Polynomial } \\
\text { Kernel functions }\end{array}$} \\
\cline { 3 - 5 } $\mathbf{( 4 : 1 )}^{*}$ & & $\mathbf{1}$ degree & 2 degree & 3 degree \\
\hline $\mathbf{9 x 9}$ & 96.8 & $99.2(98.4)$ & $97.6(96.8)$ & $95.2(91.2)$ \\
\hline $\mathbf{7 x 7}$ & 98.4 & $100(99.2)$ & $97.6(99.2)$ & $96(96)$ \\
\hline $\mathbf{5 x 5}$ & 100 & $100(99.2)$ & $97.6(99.2)$ & $96(96.8)$ \\
\hline$(3: 2)^{*}$ & & & & \\
\hline $\mathbf{9 x 9}$ & 97.6 & $97.2(95.2)$ & $93.2(93.2)$ & $89.2(87.6)$ \\
\hline $\mathbf{7 x 7}$ & 98.4 & $98.8(95.6)$ & $95.6(94)$ & $92.4(89.2)$ \\
\hline $\mathbf{5 x 5}$ & 99.2 & $99.2(95.6)$ & $96.4(93.2)$ & $92.8(90)$ \\
\hline & & & & \\
\hline
\end{tabular}

Table 4. Fuzzy Feature on IITD database

\begin{tabular}{|l|c|l|l|l|}
\hline $\begin{array}{c}\text { window } \\
\text { size }\end{array}$ & \multirow{2}{*}{ ED } & \multicolumn{3}{|c|}{$\begin{array}{c}\text { SVM using different Polynomial } \\
\text { Kernel functions }\end{array}$} \\
\cline { 1 - 4 }$(\mathbf{4 : 1})^{*}$ & & 1 degree & 2 degree & 3 degree \\
\hline $\mathbf{9 x 9}$ & 99.2 & $99.2(99.2)$ & $99.2(99.2)$ & $99.2(99.2)$ \\
\hline $\mathbf{7 x 7}$ & 100 & $99.2(99.2)$ & $99.2(99.2)$ & $99.2(99.2)$ \\
\hline $\mathbf{5 x 5}$ & 100 & $99.2(99.2)$ & $99.2(99.2)$ & $99.2(99.2)$ \\
\hline$(3: 2)^{*}$ & & & & \\
\hline $\mathbf{9 x 9}$ & 99.2 & $98.8(98.4)$ & $98.8(98.4)$ & $98.8(98.4)$ \\
\hline $\mathbf{7 x 7}$ & 99.2 & $98.8(98.4)$ & $98.8(98.4)$ & $98.8(98.4)$ \\
\hline $\mathbf{5 x 5}$ & 98.4 & $98.8(98.4)$ & $98.8(98.4)$ & $98.8(98.4)$ \\
\hline
\end{tabular}

Table 5. Sigmoid Feature on IITD database

\begin{tabular}{|l|c|c|c|c|}
\hline \multirow{2}{*}{$\begin{array}{c}\text { window } \\
\text { size }\end{array}$} & \multirow{2}{*}{ ED } & \multicolumn{4}{|c|}{$\begin{array}{c}\text { SVM using different Polynomial } \\
\text { Kernel functions }\end{array}$} \\
\cline { 3 - 5 }$(\mathbf{4 : 1})^{*}$ & & 1 degree & 2 degree & 3 degree \\
\hline $\mathbf{9 x 9}$ & 100 & 100 & 100 & 100 \\
\hline $\mathbf{7 x 7}$ & 100 & 100 & 100 & 100 \\
\hline $\mathbf{5 x 5}$ & 100 & 100 & 100 & 100 \\
\hline$(3: 2)^{*}$ & & & & \\
\hline $\mathbf{9 x 9}$ & 100 & 100 & 99.6 & 99.2 \\
\hline $\mathbf{7 x 7}$ & 100 & 100 & 99.6 & 99.2 \\
\hline $\mathbf{5 x 5}$ & 100 & 100 & 99.6 & 99.2 \\
\hline
\end{tabular}

Table 6. LBP Feature on IITD database

\begin{tabular}{|l|l|l|l|l|}
\hline \multirow{2}{*}{$\begin{array}{l}\text { win } \\
\text { size }\end{array}$} & \multirow{2}{*}{ ED } & \multicolumn{3}{|c|}{$\begin{array}{l}\text { SVM using different Polynomial } \\
\text { Kernel functions }\end{array}$} \\
\cline { 1 - 3 }$\left(\mathbf{4 : 1 ) ^ { * }}\right.$ & & 1 degree & 2 degree & 3 degree \\
\hline $\mathbf{9 x 9}$ & 99.19 & $96.7(95.96)$ & $96.7(95.9)$ & $96.77(95.9)$ \\
\hline $\mathbf{7 x 7}$ & 100 & $98.3(99.19)$ & $98.3(99.2)$ & $98.38(99.2)$ \\
\hline $\mathbf{5 x 5}$ & 100 & $100(99.19)$ & $100(99.2)$ & $100(99.1)$ \\
\hline$(3: 2)$ & & & & \\
\hline $\mathbf{9 x 9}$ & 95.16 & $88.7(90.72)$ & $88.7(90.7)$ & $88.30(90.7)$ \\
\hline $\mathbf{7 x 7}$ & 100 & $96.7(98.38)$ & $96.7(98.3)$ & $96.77(98.3)$ \\
\hline $\mathbf{5 x 5}$ & 100 & $99.5(99.19)$ & $99.2(99.2)$ & $99.1(99.2)$ \\
\hline
\end{tabular}

Table 7. Wavelet Feature (S4) on PolyU database

\begin{tabular}{|c|c|c|l|l|}
\hline \multirow{2}{*}{$\begin{array}{l}\text { win } \\
\text { size }\end{array}$} & \multirow{2}{*}{ ED } & \multicolumn{3}{|c|}{ SVM using different Polynomial Kernel } \\
functions \\
\cline { 1 - 3 }$(\mathbf{4 : 1})^{*}$ & & 1 degree & 2 degree & 3 degree \\
\hline $\mathbf{7 x 7}$ & 97.24 & $99.17(97.7)$ & $97.24(95.86)$ & $95.86(93.6)$ \\
\hline $\mathbf{5 x 5}$ & 98.89 & $100(99.4)$ & $98.34(98.07)$ & $96.96(95.8)$ \\
\hline$(3: 2)^{*}$ & & & & \\
\hline $\mathbf{7 x 7}$ & 96 & $96.69(98.2)$ & $94.07(95.04)$ & $91.32(92.2)$ \\
\hline $\mathbf{5 x 5}$ & 98.07 & $98.62(98.6)$ & $97.10(97.24)$ & $94.9(93.93)$ \\
\hline
\end{tabular}

Table 8. Wavelet Feature (S3) on PolyU database

\begin{tabular}{|l|l|l|l|l|}
\hline \multirow{2}{*}{$\begin{array}{l}\text { win } \\
\text { size }\end{array}$} & \multirow{2}{*}{ ED } & \multicolumn{3}{|c|}{ SVM using different Polynomial Kernel } \\
functions \\
\cline { 4 - 5 }$(\mathbf{4 : 1})^{*}$ & & 1 degree & 2 degree & 3 degree \\
\hline $\mathbf{9 x 9}$ & 93.93 & $97.79(97.24)$ & $95.04(94.7)$ & $91.73(90.6)$ \\
\hline $\mathbf{7 x 7}$ & 97.24 & $98.62(97.24)$ & $96.4(96.14)$ & $95.04(93.6)$ \\
\hline $\mathbf{5 x 5}$ & 97.79 & $100(99.44)$ & $98.3(98.07)$ & $96.6(95.59)$ \\
\hline$(3: 2)^{*}$ & & & & \\
\hline $\mathbf{9 x 9}$ & 91.59 & $95.17(95.04)$ & $91.7(91.04)$ & $88.29(86.7)$ \\
\hline $\mathbf{7 x 7}$ & 91.73 & $95.04(98.07)$ & $94.2(95.17)$ & $90.7(92.01)$ \\
\hline $\mathbf{5 x 5}$ & 98.07 & $98.48(98.62)$ & $96.8(97.24)$ & $94.76(93.6)$ \\
\hline & & & \multicolumn{3}{|l}{} \\
\hline
\end{tabular}

Table 9. Wavelet Feature (S2) on PolyU database

\begin{tabular}{|l|l|l|l|l|}
\hline \multirow{2}{*}{$\begin{array}{l}\text { win } \\
\text { size }\end{array}$} & \multirow{2}{*}{ ED } & \multicolumn{3}{|c|}{ SVM using different Polynomial Kernel } \\
functions \\
\cline { 1 - 3 }$(\mathbf{4 : 1})^{*}$ & & 1 degree & 2 degree & 3 degree \\
\hline $\mathbf{9 x 9}$ & 93.93 & $97.79(96.9)$ & $92.01(94.2)$ & $92.01(90.6)$ \\
\hline $\mathbf{7 x 7}$ & 96.69 & $98.6(98.3)$ & $96.4(96.4)$ & $95.04(93.3)$ \\
\hline $\mathbf{5 x 5}$ & 98.62 & $100(99.44)$ & $98.6(98.07)$ & $96.6(95.04)$ \\
\hline$(3: 2)^{*}$ & & & & \\
\hline $\mathbf{9 x 9}$ & 91.04 & $94.09(94.90)$ & $88.29(89.9)$ & $88.29(86.7)$ \\
\hline $\mathbf{7 x 7}$ & 5.45 & $97.52(97.93)$ & $94.2(94.07)$ & $90.77(91.3)$ \\
\hline $\mathbf{5 x 5}$ & 97.93 & $98.48(98.34)$ & $96.83(96.5)$ & $94.35(93.1)$ \\
\hline
\end{tabular}


Table 10.Fuzzy Feature on PolyU database

\begin{tabular}{|l|l|l|l|l|}
\hline \multirow{2}{*}{$\begin{array}{c}\text { win } \\
\text { size }\end{array}$} & \multirow{2}{*}{ ED } & \multicolumn{3}{|c|}{ SVM using different Polynomial Kernel } \\
functions \\
\cline { 1 - 3 }$(\mathbf{4 : 1})^{*}$ & & 1 degree & 2 degree & 3 degree \\
\hline $\mathbf{9 x 9}$ & 96.14 & $100(100)$ & $100(100)$ & $100(99.58)$ \\
\hline $\mathbf{7 x 7}$ & 96.41 & $100(100)$ & $100(100)$ & $100(99.72)$ \\
\hline$(3: 2)^{*}$ & & & & \\
\hline $\mathbf{9 x 9}$ & 99.03 & $99.86(99.58)$ & $99.8(99.58)$ & $99.8(99.58)$ \\
\hline $\mathbf{7 x 7}$ & 99.17 & $99.8(99.58))$ & $99.86(99.7)$ & $99.86(99.7)$ \\
\hline
\end{tabular}

Table 11.Sigmoid Feature on PolyU database

\begin{tabular}{|l|l|l|l|l|}
\hline \multirow{2}{*}{$\begin{array}{c}\text { win } \\
\text { size }\end{array}$} & \multirow{2}{*}{ ED } & \multicolumn{3}{|c|}{ SVM using different Polynomial Kernel } \\
functions \\
\cline { 1 - 3 }$(\mathbf{4 : 1})^{*}$ & & 1 degree & 2 degree & 3 degree \\
\hline $\mathbf{9 x 9}$ & 99.72 & 99.72 & 100 & 100 \\
\hline $\mathbf{7 x 7}$ & 99.72 & 99.72 & 100 & 100 \\
\hline$(3: 2)^{*}$ & & & & \\
\hline $\mathbf{9 x 9}$ & 98.89 & 99.17 & 99.03 & 98.76 \\
\hline $\mathbf{7 x 7}$ & 99.17 & 99.17 & 99.03 & 98.89 \\
\hline
\end{tabular}

Table 12.LBP Feature on PolyU database

\begin{tabular}{|l|c|l|l|l|}
\hline \multirow{2}{*}{$\begin{array}{l}\text { win } \\
\text { size }\end{array}$} & \multirow{2}{*}{ ED } & \multicolumn{3}{|c|}{ SVM using different Polynomial Kernel } \\
functions \\
\cline { 3 - 5 }$(\mathbf{4 : 1})^{*}$ & & 1 degree & 2 degree & 3 degree \\
\hline $\mathbf{9 x 9}$ & 100 & $95.59(95.3)$ & $95.3(95.04)$ & $95.3(95.04)$ \\
\hline $\mathbf{7 x 7}$ & 99.8 & $99.72(99.1)$ & $99.7(99.2)$ & $99.7(99.1)$ \\
\hline $\mathbf{5 x 5}$ & 100 & $100(100)$ & $99.8(100)$ & $99.7(100)$ \\
\hline$(3: 2)^{*}$ & & & & \\
\hline $\mathbf{9 x 9}$ & 98.28 & $93.11(86.9)$ & $93.6(86.77)$ & $93.1(86.7)$ \\
\hline $\mathbf{7 x 7}$ & 99.7 & $99.03(98.1)$ & $99.03(98.1)$ & $98.9(98.1)$ \\
\hline $\mathbf{5 x 5}$ & 99.8 & $99.86(99.7)$ & $99.8(99.58)$ & $99.5(99.58)$ \\
\hline
\end{tabular}

*(TRAINING: TESTING) SAMPLE RATIO

The fuzzy features yield $100 \%$ recognition rate on both IITD and PolyU databases. Sigmoid features give an authentication rate of $99.72 \%$ on PolyU database and $100 \%$ on IITD database on the window size $9 \mathrm{x} 9$ with the Euclidean classifier.

But the accuracy is increased to $100 \%$ on PolyU database by using SVM classifier. LBP also yields $100 \%$ recognition rate on both the databases IITD and PolyU databases for the window size of $7 \times 7$ as can be seen in Tables 6 and 12 .

We now show the plots of fuzzy features extracted from all windows belonging to samples of two different users in Fig. 7 and two samples belonging to the same user in Fig. 8 to get an idea of how they differ. The differences between the features of two different users and the same users are depicted in Fig. 9. It can be clearly seen that the differences are accentuated when the users are different as against the differences between the features of the same users. The effectiveness of the features can be judged from the relative differences.

It may be noted that our best results are due to fuzzy features whereas the best results are shown on Gabor filter in [6]. Figures 10 and 11 depict ROCs of fuzzy features for two compositions of training and test samples on PolyU and IITD databases respectively. We have got GAR of $97.5 \%$ at FAR of 0.001 for the training to test samples ratio of 5:1 for each user while [6] reports GAR of $98 \%$ at FAR of $0.04 \%$ by matching the test sample with all other palmprints. The enhanced results involving GAR of $98.4 \%$ at FAR of $3 \times 10-6 \%$ are reported in [17] using competitive coding. However we get $100 \%$ identification results using SVM as shown in Table 10. The simplicity of the proposed features especially fuzzy and sigmoid is an added advantage.

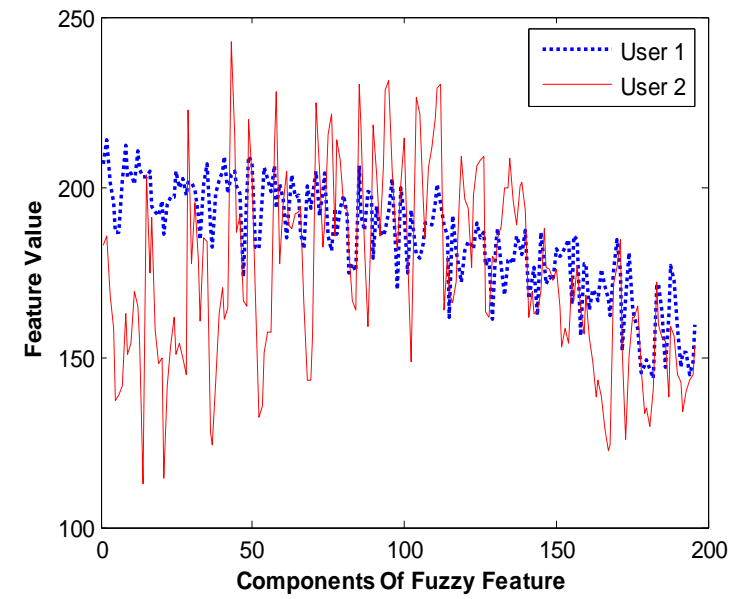

Fig 7: Fuzzy Feature plot of the palmprints of two users on PolyU database

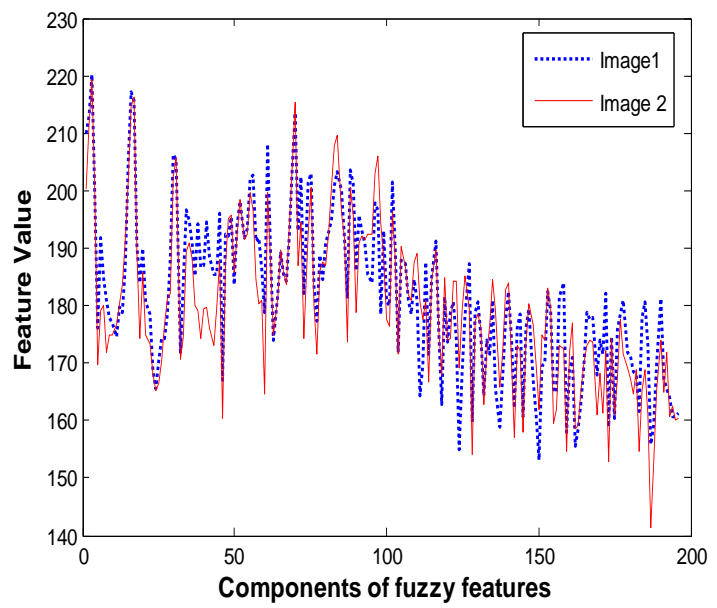

Fig 8: Fuzzy Feature plot of two palm from the same user on PolyU database

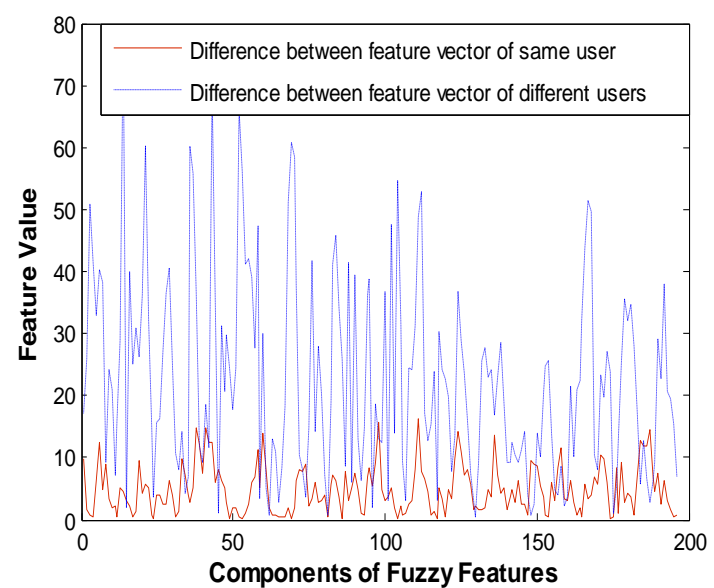

Fig 9: The difference of Fuzzy feature vector in Fig 7 and Fig 8 on PolyU database 


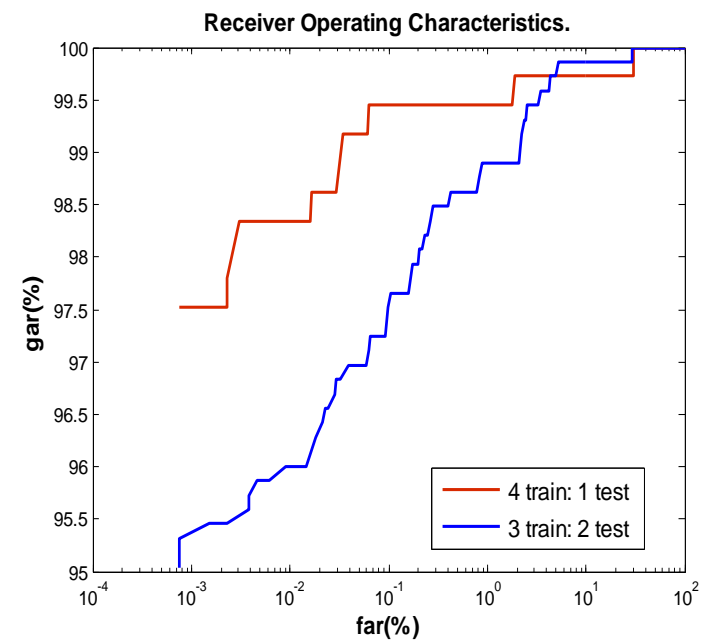

Fig 10: ROC plot of two compositions of training and testing samples using fuzzy feature on the window size of (5x5) with PolyU data base.

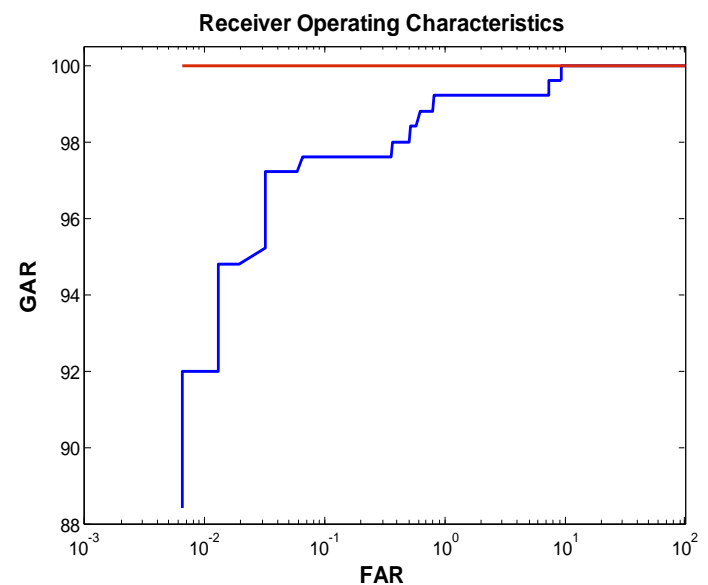

Fig 11: ROC plot of two compositions of training and testing samples using fuzzy feature with window size $(5 \times 5)$ pixels with IITD data base.

\section{CONCLUSIONS}

An experiment study of some new features is carried out on the palmprints for the authentication of users from two databases: PolyU and IITD. The feature extraction methods employed in this work confirm the effectiveness of the features obtained for the palmprint based authentication.

The recognition scores of $100 \%$ accuracy are achieved with fuzzy features as well as sigmoid features on both the databases using SVM classifier. In terms of accuracy and computational complexity, the fuzzy features from ROIs of palmprints are found to be most suitable for the authentication among all the feature types considered.

Irrespective of the type of features used, there is a marked difference in the results obtained using the Euclidean distance classifier and those with SVM classifier. SVM classifier with linear kernel function and two polynomial kernels of degrees 2 and 3 perform with an accuracy of $100 \%$ recognition score.

The main problem for achieving good authentication rates is the choice number of samples for the training and the testing. We have made several experiments by varying these numbers. It is observed that as the training samples increase the matching scores increase but as the number of testing samples increase the matching scores decrease correspondingly. The cross validation has also been done and the results are almost the same.

The main contribution of the paper is the proposition of new features and evaluation on two large databases. It may be noted that the features are so effective that even the results of simple Euclidean distance classifier are comparable to those of the complex SVM classifier. This is the reason we haven't explored other classifiers in the literature for the palmprint based authentication.

The future work will be concerned with developing new features for other modalities like hand geometry for the fusion of more than one modality for better authentication.

\section{ACKNOWLEDGMENTS}

Our thanks to the unknown reviewers for their valuable comments, also to Mr. Lei Zhang, Poly Technique University, Hong Kong for providing palmprint database.

\section{REFERENCES}

[1] Ying-Han Pang, Andrew TeohBeng Jin, David Ngo Chek Ling, "Palmprint based Cancelable Biometric Authentication System", Int. J. of Signal Processing, Vol.1, No.2, pp.93-99, 2004.

[2] N. Duta, A.K. Jain, and K.V. Mardia, "Matching of Palmprint," Pattern Recognition Letters, Vol.23, No. 4 pp. 477-485, 2001.

[3] Ying-Han Pang, Andrew Teoh Beng Jin, David Ngo Chek Ling, Fu San Hiew, "Palmprint Verification with Moments", WSCE, 2004.

[4] Lei Zhang, Zhenhua Guo, Zhou Wang, David Zhang, "Palmprint Verification Using Complex Wavelet Transform", IEEE Intl. Conf. on Image Processing, San Antonio, TX, Sept.16-19,2007.

[5] Li Fang, Maylor K.H. Leung, Tejas Shikhare, Victor Chan, Kean FattChoon, "Palmprint Classification", IEEE Int. Conf. on Systems, Manand Cybernetics, Vol. 4, 8-11 October 2006, pp.2965-2969.

[6] David Zhang, Wai-Kin Kong, Jane You, and Michael Wong, "Online Palmprint Identification", IEEE Trans. Pattern Analysis \&Machine Intelligence, Vol. 25, No. 9, pp. 1041 - 1050,September 2003.

[7] M. Hanmandlu, H.M. Gupta, Neha Mittal, and S. Vasikarla, "An Authentication System Based on Palmprint", in Proc. ITNG, IEEE Computer Society, 2009, pp.399-404.

[8] Madasu Hanmandlu, Ritu Vijay, Neha Mittal, "A Study of Some New Features for Palmprint Authentication", Proceedings of The World Congress on Engineering, WCE-2011, 6-8 July, 2011, London, U.K., pp 16231628.

[9] E. Boonchien, W. Boonchieng, and R. Kanjanavani, "Edge-Detection and Segmentation Methods for TwoDimensional Echocardiograms", Proc. Int'] Conf. Computers in Cardiology, pp. 541-544, September 2004. 
[10] T. Ojala, M. Pietikainen, and T. Maenpaa, "Multiresolution Gray-Scale and Rotation Invariant Texture Classification with Local Binary Patterns", IEEE Trans. on Pattern Analysis and Machine Intelligence, vol. 24, pp. 469-481, July 2002.

[11] Michael Goh Kah Ong, Connie Tee and Andrew TeohBeng Jin, "Touch-less Palm-print Biometric System”, in Proc.VISIAPP'08, 2008, pp. 423-430.

[12] V.N. Vapnik, Statistical Learning Theory. New York: Wiley-Interscience, 1998.

[13] B. Scholkopf, C.J.C. Burges, and A.J. Smola, Advances in kemel Methods-Support Vector Learning. Cambridge, MA: MIT Press, 1998.

[14] H. Li, Y. Liang, and Q. Xu, "Support Vector Machines and its Applications in Chemistry", Chemometrics and Intelligent Laboratory Systems, vol. 95, pp. 188-198, Feb. 2009.
[15] Chih-Chung Chang and Chih-Jen Lin, LIBSVM: a library for support vector machines, 2001. http://www.csie.ntu.edu.tw/ cjlin/libsvm

[16] H. K. Polytechnic University, "Palmprint database", Biometric Research Center Website. 2005. http://www4.comp.polyu.edu.hk/ biometrics/

[17] A. Kong and D. Zhang, "Competitive coding scheme for palmprint verification," in Proc. Int. Conf. Pattern Recog., 2004, pp. 520-523, Cambride, UK.

[18] Xiang-Qian Wu, Kuan-Quan Wang, David Zhang, "Wavelet Based Palmprint Recognition", First international conference on Machine learning and Cybernetics, Beijing, pp-1253-1257, 4-5 Nov 2002.

[19] Xian-Qian Wu, Kuan-Quan Wang, David Zhang, "Wavelet based Palmprint Recognition", Proceedings of the first international conference on machine learning and cybernetics, Beijing, 4-5 November 2002. 